\title{
INTENSIDADE DE EXERCÍCIO FÍSICO E IMUNOMODULAÇÃO: IMPACTOS EM INFECÇÕES DAS VIAS AÉREAS
}

Diego Patrick Soares Lopes
Igor Pereira Ribeiro Muniz

Universidade Federal da Bahia (UFBA), Brasil.

\section{Robson Amaro Augusto da Silva}

Núcleo de Biointegração, Instituto Multidisciplinar em Saúde, Universidade Federal da Bahia (UFBA), Brasil.

E-mail: robson.amaro@gmail.com
RESUMO: A atividade física tem sido utilizada como ferramenta de auxílio para a diminuição da incidência de diversos riscos, tais como obesidade, desenvolvimento de doenças cardiovasculares e diabetes. Além disso, ela tem sido descrita como medida imunomodulatória. $\mathrm{O}$ exercício físico possui algumas variáveis, dentre elas a frequência do exercício e a intensidade do mesmo. As variáveis nesse âmbito têm sido relacionadas a diferentes efeitos na modulação da resposta desenvolvida em diferentes sistemas e por vezes pode estar associada ao aumento da suscetibilidade a infecções ou ao aumento da resistência a infecções. O sistema respiratório representa uma das maiores portas de entrada para microrganismos patogênicos apresentando grande incidência de doenças infecciosas em atletas de elite. O exercício moderado tem sido associado a uma proteção contra infecções respiratórias causadas por vírus e por bactérias quando comparado ao exercício intenso, que de forma contrária, tem sido relacionado ao aumento da suscetibilidade a essas infecções principalmente em atletas praticantes de atividade física intensa. Nessa revisão pontuamos os aspectos mais recentes associados à modulação da resposta imune pela atividade física e suas implicações em infecções no sistema respiratório.

PALAVRAS-CHAVE: Exercício; Imunologia; Microbiologia; Respiração.

\section{INTENSITY OF PHYSICAL EXERCISES AND IMMUNOMODULATION: IMPACTS IN INFECTIONS OF THE AERIAL PATHWAYS}

\begin{abstract}
Physical activity has been employed as a tool to lessen the occurrence of several types of risks, such as obesity, development of cardiovascular diseases and diabetes. In fact, it has been described as an immunomodulating measure. Physical exercise has several variables among which may be mentioned frequency of exercises and their intensity. Variables have been related to different effects in the modulation of response developed in several systems and frequently due to its association to increase in susceptibility to infections or to increase in the resistance to infections. The respiratory system is one of the biggest entrances for pathogenic microorganisms with the occurrence of infectious diseases in athletes. Moderate exercises have been associated to the protection against respiratory infections caused by viruses and bacteria when it is compared to intense exercises; otherwise, it is related to increase in susceptibility to these infections mainly in athletes who practice intense physical activity. Current review demonstrates the most recent aspects associated to the modulation of immune response by physical activity and its implications in the infections of the respiratory system.
\end{abstract}

KEY WORDS: Breathing Exercises; Immunology; Microbiology; Breathing. 


\section{INTRODUÇÃO}

A atividade física é conhecida por melhorar parâmetros fisiológicos como perda de peso, redução no risco de desenvolvimento de doenças cardiovasculares e controle glicêmico (HASKELL et al., 2007; LLOPIS; REIG GARCÍA-GALBIS, 2015). Entre outros benefícios obtidos com a prática da atividade física monitorada tem-se, também, a melhora de parâmetros imunológicos (EDSFELDT et al., 2015; VAN CRAENENBROECK et al., 2014).

Os benefícios descritos na literatura para as atividades abrangem outros pontos como prevenção de doenças secundárias ao sedentarismo, tais como hipertensão primária e risco no desenvolvimento de câncer de mama (DHURANDHAR; KEITH, 2014; FEBER; NIEMIRSKA, 2015; HASKELL et al., 2007; LLOPIS; REIG GARCÍA-GALBIS, 2015; NEILSON et al., 2009). Outro ponto importante com relação à prática de atividades físicas regulares se dá pela manutenção dos níveis glicêmicos basais e melhora tanto na captação quanto no uso da glicose pelos tecidos metabólicos (HIRABARA et al., 2012; HYVÖNEN; SPALDING, 2014; MCLELLAN et al., 2007).

A prática de exercício físico regular é responsável pordiminuiroriscodeinfecçõesviraisnotratorespiratório. Opostamente à prática de exercícios físicos intensos, praticados por atletas, provocam diversas alterações neuroendócrinas, bioquímicas e cardiovasculares, o que contribui para distúrbios no sistema imune (MARTÍNEZ, ALFREDO CÓRDOVA; ALVAREZĐMON, 1999). As infecções do trato respiratório são responsáveis por um alto índice de internações e consequentes óbitos (MISHARIN; CHI; BUDINGER, 2015; RANTALA et al., 2015). Essas infecções podem direcionar para um perfil inflamatório específico, de maneira crônica, como o que ocorre na doença pulmonar obstrutiva crônica, o que pode induzir o aparecimento de infecções concomitantes (BEASLEY et al., 2012). Dentre os problemas que estas infecções podem acarretar estão declínio da capacidade respiratória, capacidade de exercício e do status de saúde (BEASLEY et al., 2012).

Como sabido, o exercício físico regular ajuda na manutenção da função e da força muscular além de melhorar a atividade cardiorrespiratória e qualidade de vida (LEEUWEN et al., 2013). A intensidade e o tempo de atividade física influenciam de maneira direta no comportamento do corpo. Muitos estudos têm relacionado o exercício físico, em intensidade moderada, como possível terapia para algumas situações de riscos (LEEUWEN et al., 2013).

Em contrapartida, o exercício físico intenso tem sido relacionado com o aumento da incidência do risco de infecções no trato respiratório (KUROWSKI et al., 2014a). Dessa maneira, esta revisão visa trazer aspectos importantes sobre os efeitos imunomodulatórios do exercício físico e suas implicações em infecções do sistema respiratório.

\section{ATIVIDADE FÍSICA COMO PRÁTICA DE SAÚDE}

A atividade física traz inúmeras melhorias para seus praticantes, tanto nos parâmetros fisiológicos como psicológicos. Indivíduos que praticam atividades físicas apresentam menores incidências de doenças e uma melhor percepção para a qualidade de vida (SAGUN et al., 2014). O exercício atua na redução da massa corporal, diminui a resistência à insulina, além de estimular a utilização de substratos metabólicos (RINGSEIS; MOOREN; KRÜGER, 2015). Praticantes de exercícios físicos apresentam uma melhora na regulação da pressão arterial por alterações hemodinâmicas e neuro-humorais (RONDON; BRUM, 2003).

Com relação a parâmetros bioquímicos, a atividade física reduz a produção de lipoproteínas de baixa densidade (LDL) e promove redução da glicose sérica em jejum, além do aumento das lipoproteínas de alta densidade (NISHIDA et al., 2015; PARRA-SÁNCHEZ et al., 2015). As mudanças, tanto no perfil lipídico quanto no perfil glicêmico, são de grande importância, uma vez que estes estão relacionados com o desenvolvimento de aterosclerose e síndromes metabólicas como é o caso da diabetes mellitus (GLEESON et al., 2011; LLOPIS; REIG GARCÍA-GALBIS, 2015).

O hábito da prática regular de atividades físicas reduz o risco de comorbidades associadas ao aumento de peso. Dentre essas comorbidades têm-se 
o desenvolvimento de cálculos biliares, alterações na microbiota intestinal com prevalência de Firmicutes e Bacteriodetes na obesidade além de maior suscetibilidade a infecções (DHURANDHAR; KEITH, 2014). A atividade física também pode atuar como ferramenta imunomoduladora. Estudos como o desenvolvido por Edsfeldt et al. (2015) relacionam a prática de exercício com a redução na produção de TNF- $\alpha$. Essa diminuição foi relacionada com o menor desenvolvimento de placas de ateroma (EDSFELDT et al., 2015; PARRA-SÁNCHEZ et al., 2015).

A produção de citocinas anti-inflamatórias, como a interleucina-10 (IL-10) e IL-1ra, é bem documentada em indivíduos praticantes de exercícios físicos, em contrapartida, a prática regular de exercícios reduz a produção de citocinas pró-inflamatórias como o fator de necrose tumoral alfa (TNF- $\alpha$ ) e IL-1 $\beta$ (EDSFELDT et al., 2015; PARRA-SÁNCHEZ et al., 2015). A atividade física tem atuado como um importante promotor de saúde e ferramenta para uma melhor percepção de qualidade de vida. Desta maneira, a relação entre a prática de atividade física e os efeitos imunomoduladores tem sido estudada.

\section{A ATIVIDADE FÍSICA COMO FERRAMENTA IMU- NOMODULADORA}

Nas últimas décadas, tem sido apontado que o exercício físico aeróbico nas intensidades moderada e intensa modula o sistema imunológico dos indivíduos que o praticam. A atividade física apresenta tanto benefícios como malefícios, a depender da sua intensidade, tempo de atividade e tipo de atividade. A redução na expressão de receptores como Toll Like Receptors (TLRs) é um dos possíveis mecanismos, tanto em exercício físico moderado quanto intenso. Estudos têm observado que com a prática de exercício, a expressão de TLRs dos tipos $1,2,3$ e 4, além de moléculas como MHC do tipo 2 e proteínas co-estimuladoras como CD-80 e CD-86, tem sido diminuída. Essas modificações caracterizam um efeito anti-inflamatório do exercício (CHEN et al., 2013; FERNANDEZ-GONZALO et al., 2014; FLYNN; MCFARLIN, 2006; GLEESON et al., 2011)

O exercício físico moderado, praticado de maneira regular, tem a capacidade de induzir o músculo esquelético a atuar como um órgão endócrino, na produção de moléculas semelhantes a citocinas, chamadas de miocinas (LEEUWEN et al., 2013; MATHUR; PEDERSEN, 2008). As miocinas mais abordadas, que apresentam efeitos anti-inflamatórios durante o exercício físico, são a IL-10, o antagonista do receptor de IL-1 (IL-1Ra) e a IL-6, que é parcialmente responsável por aumentar a liberação de hormônios liberados em estados de estresse, como o cortisol (GLEESON, 2007; GLEESON et al., 2011; LEEUWEN et al., 2013; PEDERSEN, 2011).

Mudanças geradas pelo exercício moderado, de modo geral, incluem aumento na produção de hormônios, gerados em condições de estresse, aumento da resposta Th2 e diminuição na resposta Th1, o que diminui a inflamação local exacerbada (MARTIN; PENCE; WOODS, 2009). Essa resposta tem sido descrita como eficiente no controle de infecções virais no trato respiratório superior, ao contrário do que acontece na resposta gerada pelo exercício intenso.

Quanto ao exercício físico intenso, seu alto perfil anti-inflamatório pode se dar através da mudança de perfil de células T. Trabalhos como o de Wang et al. (2012) demonstram o aumento significativo de células CD-4+/CD-25+/Foxp3+ quando comparado a indivíduos não praticantes de atividade física. $\mathrm{O}$ aumento de Células T reguladoras (Treg), no sangue periférico dos indivíduos que praticaram atividade física intensa, tem sido demonstrado. Em contrapartida, indivíduos que praticaram atividade física moderada não tiveram esse aumento significativo. Essas mudanças na resposta imune adaptativa podem justificar a maior suscetibilidade a infecções em atletas de elite que praticam atividade física intensa (MORGADO et al., 2012).

Desse modo, o entendimento dos efeitos do exercício físico no sistema imune tem sido ampliado, porém ainda não são muito compreendidos. Na atualidade ainda são poucos os estudos que tentam correlacionar esses efeitos com a incidência e os impactos em infecções no trato respiratório.

\section{INFECÇÕES NO TRATO RESPIRATÓRIO}

Devido às importantes consequências clínicas, muitos estudos têm sido feitos no intuito de uma melhor 
compreensão dos mecanismos que ocorrem durante as infecções no trato respiratório (BEASLEY et al., 2012; SATURNI et al., 2015). Muitos microrganismos habitam a microbiota do trato respiratório em condições normais, como Staphylococcus epidermidis, Corynebacterium spp., Streptococcus spp. e Neisseria spp., podendo eventualmente ser colonizada por Staphylococcus aureus, no caso das vias aéreas superiores. A determinação da microbiota das vias aéreas inferiores é mais difícil por haver contaminação pelos microrganismos das vias superiores (AUSTRIAN, 1968).

Indivíduos que possuam condições como a doença pulmonar obstrutiva crônica (DPOC) sofrem alterações em sua microbiota do trato respiratório. As mudanças mais encontradas durante os exames é a presença de microrganismos como Haemophilus influenzae, Streptococcus pneumoniae e Moraxella catarrhalis. A gravidade da doença pode favorecer o aparecimento de outros microrganismos como Pseudomonas aeruginosa, Haemophilus parainfluenzae e Staphylococcus aureus (BEASLEY et al., 2012; PARK et al., 2014).

Existe um extenso número de patógenos virais e bacterianos capazes de gerar infecções no trato respiratório. O vírus sincicial respiratório (VSR) é um dos diversos patógenos responsáveis por causar infecções no trato respiratório. As infecções geradas por esse vírus apresentam alto índice de óbitos e internações, sendo a população infantil a mais afetada (VANDINI et al., 2015). Infecções virais também apresentam grande relevância como as infecções causadas pelo vírus Influenza tipo A (VIA), que no início do século 20 causou uma pandemia com a morte de 50 a 100 milhões de pessoas (MISHARIN; CHI; BUDINGER, 2015).

\subsection{RESPOSTA IMUNE DESENVOLVIDA FRENTE ÀS INFECÇÕES NO TRATO RESPIRATÓRIO}

Hojeestábemestabelecidoqueotrato respiratório representa uma grande porta de entrada para diversos patógenos e outras partículas, sendo altamente suscetível a infecções e processos inflamatórios. Muitos estudos têm sido realizados visando uma melhor compreensão sobre o processo de inflamação gerado nesses processos
(LECAILLE; LALMANACH; ANDRAULT, 2015; LIU et al., 2011; MESSAGE; JOHNSTON, 2004). Com a existência de diversas patologias no trato respiratório causadas por infecções, as estruturas do trato respiratório desenvolvem respostas de defesa. Essas respostas são organizadas e realizadas pelo sistema imune.

As vias aéreas apresentam uma ampla defesa contra partículas inaladas durante a inspiração, dificultando o estabelecimento da infecção por diversos patógenos. O muco, as células epiteliais, as junções intercelulares promovem a limpeza e a barreira necessária contra partículas estranhas e microrganismos diversos inalados juntamente com o ar (LECAILLE; LALMANACH; ANDRAULT, 2015). Outra linha de defesa ocorre através da organização e ação de células coordenadas pelo sistema imune através do processo inflamatório. A presença de microrganismos gera uma resposta direcionada e bem desenvolvida. Nessa resposta, as células do sistema imune se deslocam para o sítio de infecção, produzem citocinas, quimiocinas e imunoglobulinas específicas para o controle de invasores potencialmente lesivos (LIU et al., 2011).

O processo inflamatório gerado no controle desses patógenos ocorre através da combinação da resposta inata e a resposta imune adaptativa. Os elementos da resposta inata incluem fagócitos como neutrófilos e macrófagos, células natural killers (NK), mastócitos, basófilos e células epiteliais. Estas células promovem a liberação de citocinas, defensinas e proteínas surfactantes, que participam da neutralização de patógenos (MESSAGE; JOHNSTON, 2004). Em uma infecção viral primária, de modo geral, os vírus se multiplicam no trato respiratório e, em aproximadamente dois dias, os níveis de IFN do tipo I são aumentados. O IFN do tipo I atua ativando células NK que irão eliminar as células infectadas e liberar mais citocinas, como o IFNque irá ativar e potencializar a ação de macrófagos nas vias aéreas (MESSAGE; JOHNSTON, 2004).

O estudo de Amineva e Gern (2003) demonstra a suscetibilidade dos organismos a infecções pelo Rhinovirus, que cliva e inativa moléculas do sistema complemento, como C3 e C5, que estão associadas com a destruição de células infectadas pelo Rhinovirus. A ausência da molécula C3 também pode aumentar 
a suscetibilidade de infecções causadas pelo vírus Influenza. Animais com deficiência na produção dessa molécula apresentam alta incidência de infecções primárias pelo vírus Influenza. A ausência dessa molécula reduz a drenagem de células para o linfonodo e menor recrutamento de células $\mathrm{T}$ efetoras $\mathrm{CD} 4+$ e CD8+ produtoras de interferon-gama (IFN- $\gamma$ ) (KOPF et al., 2002; ANDERS et al., 1994).

O estudo de Lee, Luchian e Park (2015) demonstrou a relevância do exercício físico no controle de pneumonia causada por Staphylococcus aureus, no qual o exercício regular e moderado foi capaz de modular positivamente a produção de citocinas como TNF- $\alpha$ e IL1- $\beta$ e aumento na produção de oxido nítrico (NO), gerando um ambiente altamente pró-inflamatório e também aumentando a expressão, por macrófagos residentes, de peptídeos antimicrobianos como catelicidinas.

A resposta imune adquirida envolve a produção de anticorpos e ativação de células T citotóxicas através do processamento e apresentação de antígenos pelas células dendríticas (DC) (MESSAGE; JOHNSTON, 2004). $\mathrm{O}$ recrutamento de células T é dependente da produção de quimiocinas e expressão de moléculas de adesão no endotélio de tecidos que estejam passando pelo processo inflamatório. A produção de imunoglobulinas (Igs) por células B requer tempo, os níveis de IgA são detectados em 3 dias, IgM entre 5 e 6 dias e $\operatorname{IgG}$ de 7 a 8 dias, aumentando em quantidade e atividade nas próximas 2 ou 3 semanas. Nos mecanismos imunes específicos, as células T CD8+ e as imunoglobulinas são responsáveis por controlar as infecções virais geralmente dentro de sete dias (YANG et al., 2002).

Além das respostas via sistemas complemento, celular e humoral, o organismo possui outros tipos celulares envolvidos na sua própria defesa. Células pertencentes ao trato respiratório, como as células epiteliais do trato respiratório, produzem proteínas e peptídeos antimicrobianos. Essas moléculas se apresentam como importante arma contra diferentes microrganismos patogênicos como bactérias, vírus e fungos (SHEN et al., 2014). Os mais abundantes componentes antimicrobianos do líquido de superfície das vias aéreas são as $\beta$-defensinas e outras grandes moléculas como lisozima, lactoferrina, fosfolipase $\mathrm{A}_{2} \mathrm{e}$ os inibidores da protease secretada por leucócito (IPSL) (LECAILLE; LALMANACH; ANDRAULT, 2015; MARTIN et al., 2015).

Infecções causadas pelo vírus sincicial respiratório (VSR) possuem alta incidência no trato respiratório. Em estudos, como o realizado por Vandini et al. (2015), foi demonstrado que indivíduos que apresentaram alto número de neutrófilos e monócitos, com redução no número de linfócitos, se classificaram como casos severos. No mesmo estudo foi constatado que indivíduos que possuíam predominantemente células $\mathrm{T}$ CD8+ e linfócitos B possuíram bom prognóstico.

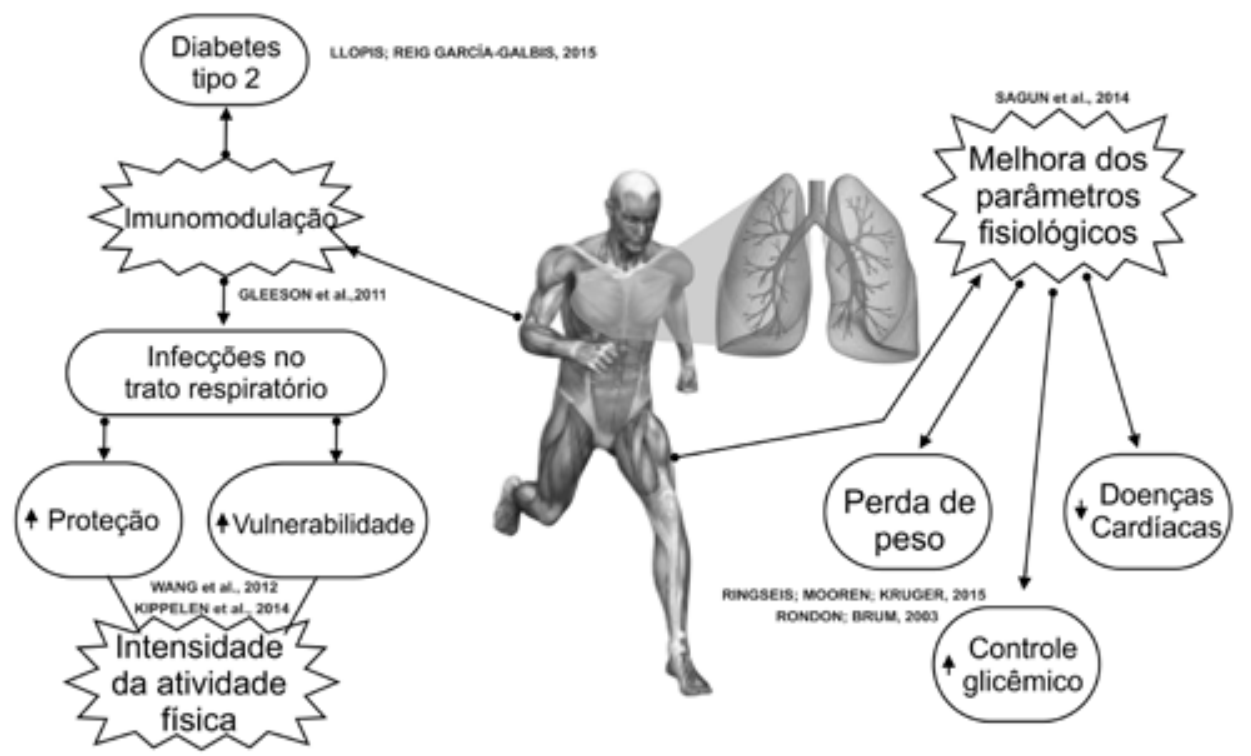

Figura 1. Esquema ilustrativo das mudanças geradas no organismo devido à prática de atividade física 


\subsection{A ATIVIDADE FÍSICA MODULANDO OS SISTEMAS ENDÓCRINO E IMUNE}

A atividade física tem sido documentada como promotora de alterações nos sistemas endócrino e imune. Quando a integridade do corpo é alterada há a ativação de vias de sinalização neuroendócrinas e os exercícios físicos atuam tanto como estressores como modificadores da resposta sistêmica. Utilizada como um confiável modelo de estresse físico, a atividade física promove modificações nas concentrações séricas de hormônios do estresse como catecolaminas (epinefrina e norepinfrina), hormônio do crescimento $(\mathrm{GH})$, cortisol e dos hormônios sexuais (testosterona e estrógeno) (GAGNON et al., 2014; KRÜGER et al., 2011; LANCASTER et al., 2004; PEDERSEN; HOFFMAN-GOETZ, 2000).

As catecolaminas, em especial a epinefrina, têm sido relacionadas com o aumento da atividade de células NK durante o exercício, favorecendo o recrutamento dessas células para o sangue sendo este efeito ligado ao fato de que essas células expressam um alto número de receptores adrenérgicos $\left(\beta_{2}\right)$ (MAISEL et al., 1990). A atividade física aumenta os níveis séricos de $\mathrm{GH}$. O GH reduz o recrutamento de linfócitos para a circulação, porém quando este é combinado com epinefrina apresentam um aumento no recrutamento de neutrófilos para a corrente sanguínea durante o estresse físico (PEDERSEN; HOFFMAN-GOETZ, 2000).

A influência do exercício físico na produção de cortisol depende da duração da atividade, sendo que, quanto maior a produção, menor a produção de IgA salivar, aumentando a suscetibilidade a infecções no trato respiratório (GILLUM et al., 2013). O aumento nas concentrações séricas tem sido encontrado quando são desenvolvidas atividades físicas de longa duração, não apresentando mudanças significativas quando em atividades de curta duração (GALBO, 1983).

Em estudo desenvolvido por Kruger et al. (2011) foi demonstrado que o treino de resistência intenso (TRI) aumenta as concentrações plasmáticas de cortisol significantemente, enquanto o treino de resistência moderado não apresenta alterações significativas na produção de cortisol. O aumento na apoptose de linfócitos se mostrou acompanhado da redução da expressão de Bcl-2, uma proteína antiapoptótica, encontrada nas membranas intracelulares, e um aumento na regulação de receptores CD95, indicando a ativação da morte celular, tem sido relacionado com o aumento da produção de cortisol (KRÜGER et al., 2011; LANCASTER et al., 2004; RISØY et al., 2003).

Em outro estudo, Hoffman-Goetz et al. (1999), ao incubar células do baço e do timo com concentrações de glicocorticoides próximas aos valores encontrados após uma atividade física intensa, observaram um aumento do número de células em apoptose após 24 horas de incubação. A apoptose de linfócitos estaria ligada a níveis de cortisol aumentados via atividade física intensa, com produção de espécies reativas de oxigênio (ROS) via danos no DNA e redução nos níveis de enzimas como a peróxido desmutase e a catalase (PEDERSEN; HOFFMANGOETZ, 2000).

A testosterona tem sua concentração sérica aumentada após atividade física de alta intensidade e curta duração, o mesmo acontece quando a intensidade deste exercício é moderada (RODRIGUES DA CONCEIÇÃO et al., 2014; ZMUDA et al., 1996; CADOUXHUDSON; FEW, 1985). O aumento de testosterona causado pela atividade física induz à redução na expressão de citocinas como IL-4, IL-5 e IFN- $\gamma$, reduzindo também os níveis de imunoglobulinas como IgM e IgG em testes in vitro (ARANEO et al., 1991; KANDA et al., 1996).

Uma relação importante existe entre a produção de testosterona e a de cortisol, a razão entre cortisol e testosterona é alterada pela prática de exercícios físicos prolongados e intensos (ALGHADIR; GABR; ALY, 2015; HEJAZI; HOSSEINI, 2012). O aumento na produção de testosterona durante o exercício físico pode modular a atividade de macrófagos e linfócitos, que possuem receptores para hormônios sexuais, podendo aumentar a suscetibilidade a infecções em atletas profissionais (GAGNON et al., 2014; HEJAZI; HOSSEINI, 2012; PEDERSEN; HOFFMAN-GOETZ, 2000).

Um estudo desenvolvido por Bini et al. (2014) demonstrou a influência da testosterona frente 0 controle da tuberculose, onde camundongos castrados apresentaram maior sobrevivência e menor carga de Mycobacterium tuberculosis. No mesmo estudo, foi verificado o aumento da produção de citocinas como 
TNF- $\alpha$, Il-12 e IFN- $\gamma$, aumentando a eficiência da resposta. Embora descrita a interação entre o sistema imune, exercício físico e infecções este tema ainda é muito controverso e carece de um melhor entendimento sobre os mecanismos exatos desta relação.

\subsection{O EXERCÍCIO FÍSICO COMO MODULADOR DO RISCO DE INCIDÊNCIA EM INFECÇÕES VIRAIS NO TRATO RESPIRATÓRIO}

A depender da intensidade do exercício físico, ao invés de adquirir parâmetros saudáveis, o organismo pode aumentar o seu risco de ser infectado. Atletas praticantes de esportes de alta intensidade, em diversos estudos, têm o seu risco de infecções no trato respiratório aumentado (GLEESON; PYNE, 2000; KIPPELEN et al., 2012; KUROWSKI et al., 2014b); enquanto isso, outros trabalhos mostram o risco diminuído dessas infecções quando a prática de atividade física é de intensidade moderada (WARREN et al., 2015).

Clínicas de medicina esportiva registraram que de $30 \mathrm{a} 40 \%$ dos atletas de elite que vão à consulta possuem sintomas sugestivos de infecções no trato respiratório superior, o que reforça o aumento do risco causado pelo exercício físico intenso (KIPPELEN et al., 2012). Alguns trabalhos indicam essa maior suscetibilidade devido a efeitos negativos do exercício na produção de algumas proteínas, tal como a proteína CC16 (KUROWSKI et al., 2014b). A proteína CC16 tem sido relacionada com a manutenção do epitélio respiratório (KUROWSKI et al., 2014a).

Outros trabalham indicam a suscetibilidade a infecções no trato respiratório devido a ações anti- inflamatórias causadas pelo exercício físico intenso. O trabalho de Wang et al. (2011) reforça essa informação, uma vez que este trabalho detecta o aumento de Células T regulatórias devido à prática de exercício físico intenso, o que gera um ambiente anti-inflamatório. Outro possível efeito anti-inflamatório é na diminuição na secreção de imunoglobulina do tipo A (IgA) da saliva, que é causada devido à prática de exercício intenso (HAYASHIDA et al., 2015). A IgA é responsável pela imobilização de antígenos e possíveis patógenos que tentem estabelecer infecções em ambientes como o trato respiratório (GLEESON; PYNE, 2000).

O exercício moderado tem sido indicado por sua capacidade de redução do risco de infecções no trato respiratório superior causadas por vírus, como Rhinovirus e Influenza (MARTIN et al., 2009). Trabalhos como os de Wang et al. (2012) e Matthews et al. (2008) detectaram relações entre frequência e intensidade do exercício na diminuição do risco de infecções no trato respiratório. Pessoas com moderada frequência na prática de atividade física, até 3 dias na semana, e intensidade moderada obtiveram menores incidências de infecções virais no trato respiratório quando comparadas com pessoas menos ativas fisicamente (CRANE-GODREAU et al., 2009; WANG et al., 2012).

Foi observado no trabalho de Ghollamnezhad et al. (2013) uma mudança no perfil de citocinas expressas em animais que praticaram exercício com intensidade moderada e intenso. Os que praticaram exercício moderado obtiveram aumento na produção de IFN- $\gamma \mathrm{e}$ diminuição na expressão dos níveis de IL-4, enquanto os animais com prática de exercício intenso apresentaram um padrão inverso. 


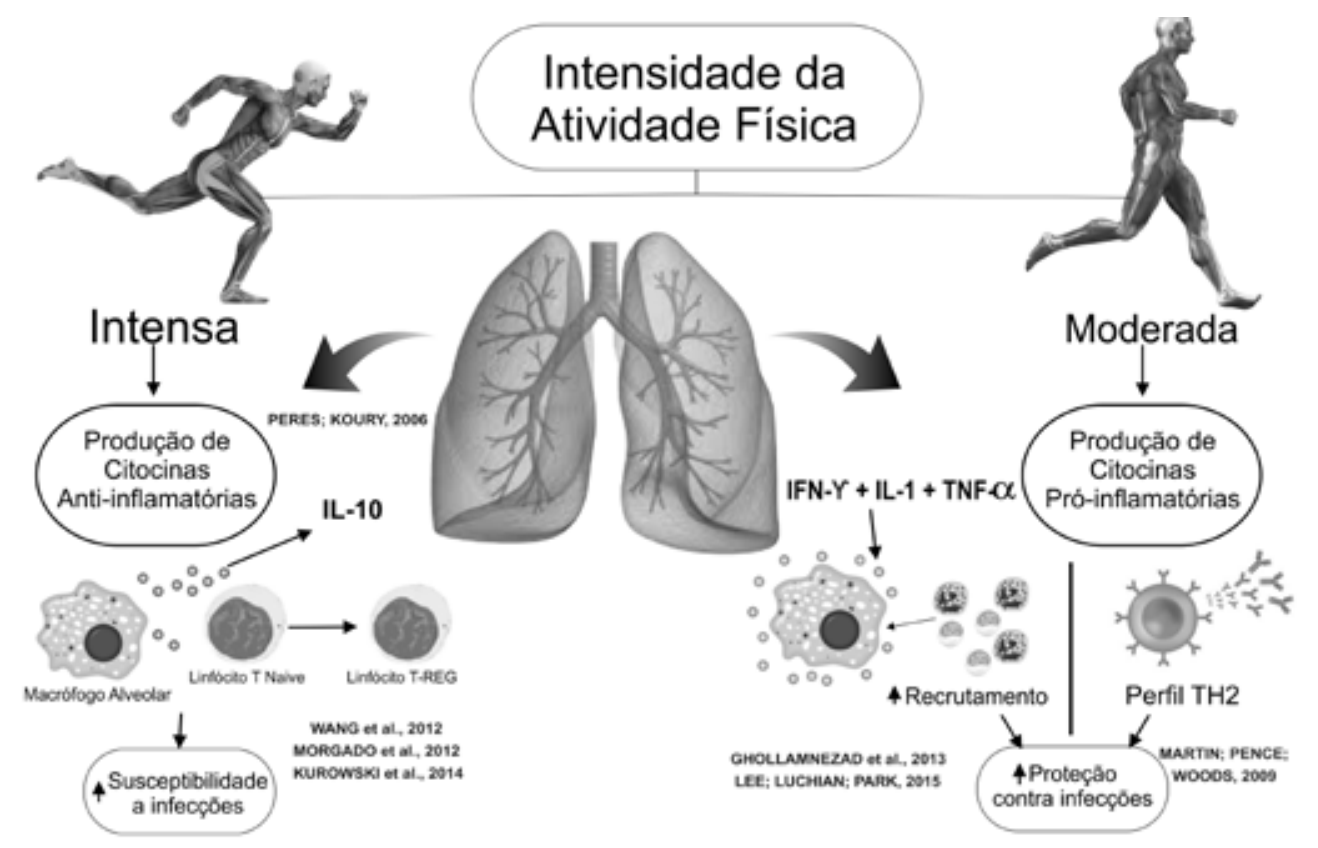

Figura 2. Esquema apontando as diferentes modulações do sistema imune de acordo com a intensidade da atividade física praticada pelo organismo

\section{CONCLUSÃO}

Diferentes questões sobre as intensidades de exercício e suas consequentes mudanças nos parâmetros imunológicos continuam sem resposta. Até o momento há um entendimento que a prática de exercício físico na intensidade moderada gera efeitos benéficos, a nível fisiológico, hormonal e imunológico, enquanto que atividades intensas apresentam alterações mais controversas. Estes fatos apontam para a necessidade de mais pesquisas acerca da influência da atividade física e infecções do trato respiratório, bem como sua modulação no eixo neuroendócrino em diferentes situações de atividades físicas.

\section{REFERÊNCIAS}

ALGHADIR, A. H.; GABR, S. A.; ALY, F. A. The effects of four weeks aerobic training on saliva cortisol and testosterone in young healthy persons. J. Phys. Ther. Sci., v. 27, p. 2029-2033, 2015.

AMINEVA, S. P.; GERN, J. E. Rhinovirus 3C protease cleaves the $\mathrm{C} 3$ and $\mathrm{C} 5$ complement factors. Am. J. Respir. Crit. Care Med., v. 167, p. A212, 2003.
ANDERS, E. M.; HARTLEY, C. A.; READING, P. C.; EZEKOWIZ, R. A. Complement-dependent neutralization of influenza virus by a serum mannose-binding lectin. $\mathbf{J}$. Gen. Virol., v. 75, p. 615-622, 1994.

ARANEO, B. A.; DOWELL, T.; DIEGEL, M.; DAYNES, R. A. Dihydrotestosterone exerts a depressive influence on the production of interleukin-4 (IL-4), IL-5, and gammainterferon, but not IL-2 byactivated murine T cells. Blood, v. 78, p. 688-699, 1991.

AUSTRIAN, R. The bacterial flora of the respiratory tract. Some knowns and unknowns. Yale J Biol Med., v. 40, n. 5-6, p. 400-13, 1968.

BEASLEY, V.; JOSHI, P. V.; SINGANAYAGAM, A.; MOLYNEAUX, P. L.; JOHNSTON, S. L.; MALLIA, P. Lung microbiology and exacerbations in COPD. Int $\mathbf{j}$ chronic obstr pulm dis., v. 7, p. 555-569, jan. 2012.

BINI, E. I.; ESPINOSA, D. M.; CASTILlO, B. M.; PAYÁN, J. B.; COLUCCI, D.; CRUZ, A. F.; ZATARAIN, Z. L.; ALGONSECA, E.; PARDO, M. R.; BOTASSO, O.; PANDO, R. H. The influence of sex steroid hormones in the immunopathology of experimental pulmonary tuberculosis. PLoS ONE, v. 9, n. 4, p. 2-10, 2014.

CADOUXHUDSON, T. A.; FEW, J. D.; IMMS, F. J. The 
effect of exercise on the production and clearance of testosterone in well trained young men. Eur J Appl Physiol 54: 321-325, 1985.

CHEN, R.; FEND, L.; RUAN, M.; LIU, X.; ADRIOUCH, S.; LIAO, H. Mechanical-Stretch of C2C12 Myoblasts Inhibits Expression of Toll-Like Receptor 3 (TLR3) and of Autoantigens Associated with Inflammatory Myopathies. PloS one, v. 8, n. 11, p. e79930, 2013.

CRANE-GODREAU, M. A.; MACCANI, M. A.; ESZTERHAS, S. K.; WARNER, S. L.; JUKOSKY, J. A.; FIERING, S. Exposure to Cigarette Smoke Disrupts CCL20-Mediated Antimicrobial Activity in Respiratory Epithelial Cells. Open Immunol J., p. 86-93, 2009.

DHURANDHAR, E. J.; KEITH, S. W. The aetiology of obesity beyond eating more and exercising less. Best Pract Res Clin Gastroenterol., v. 28, n. 4, p. 533-544, 2014.

EDSFELDT, A.; GRUFMAN, H.; ASCIUTTO, G.; NITULESCU, M.; PERSSON, A.; NILSSON, M.; NILSSON, J.; GONÇALVES, I. Circulating cytokines reflect the expression of pro-inflammatory cytokines in atherosclerotic plaques. Atherosclerosis v. 241, p. 443449, 2015.

FEBER, J.; NIEMIRSKA, A. Primary hypertension is a disease of premature vascular aging associated with neuro-immuno-metabolic abnormalities. Pediatr Nephrol, n. 1-10, 2015.

FERNANDEZ-GONZALO, R.; DE PAZ, J. A.; RODRIGUEZMIGUELEZ, P.; CUEVAS, M. J.; GONZALEZ-GALLEGO, J. TLR4-Mediated Blunting of Inflammatory Responses to Eccentric Exercise in Young Women. Mediat inflamm., v. 2014, p. 1-11, 2014.

FLYNN, M. G.; MCFARLIN, B. K. Toll-like receptor 4: link to the anti-inflammatory effects of exercise? Exerc Sport Sci Rev, v. 34, n. 4, p. 176-181, 2006.

HOLLOSZY, J. O. Hormonal and metabolic adaption to exercise. J Appl Physiol, v. 57, n. 4, 1984.

GAGNON, D. D.; GAGNON, S. S.; RINTAMAKI, H.; TORMAKANGAS, T.; PUUKA, K.; HERZIG, K. H.;
KYROLAINEN, H. The Effects of Cold Exposure on Leukocytes, Hormones and Cytokines during Acute Exercise in Humans. PLoS ONE, v. 9, n. 10, p. e110774, 2014.

GHOLAMNEZHAD, Z.; BOSKABADY, M. H.; HOSSEINI, M.; SANKIAN, M.; RAD, A. K. Evaluation of immune response after moderate and overtraining exercise in wistar rat. Iran J Basic Med Sci., v. 17, n. 1, p. 1-8, 2014.

GILLUM, T.; KUENNEN, M.; GOURLEY, C.; SCHNEIDER, S.; DOKLADNY, K.; MOSELEY, P. Salivary Antimicrobial Protein Response To Prolonged Running: a Field Based Observational Study. Biology of Sport, v. 30, n. 1, p. 3-8, 2013.

GLEESON, M. Exercise and Inflammation Immune function in sport and exercise. J. Appl. Physiol. n. 40, p. 693-699, 2007.

GLEESON, M.; BISHOP, N.; OLIVEIRA, M.; MCCAULEY, T.; TAULER, P. Sex differences in immune variables and respiratory infection incidence in an athletic population. Exerc immunol rev., v. 17, n. 0, p. 122-135, 2011.

GLEESON, M.; PYNE, D. B. Exercise effects on mucosal immunity. Immunol cell boil., v. 78, n. 5, p. 536-544, 2000.

HASKELL, W. L.; LEE, I. M.; PATE, R. R.; POWELL, K. E.; BLAIR, S. N.; FRANKLIN, B. A.; MACERA, C. A.; HEALTH, G. W.; THOMPSON, P. D.; BAUMAN, A. Physical activity and public health: Updated recommendation for adults from the American College of Sports Medicine and the American Heart Association. Med sci sports exerc., v. 39, n. 8, p. 1423-1434, 2007.

HAYASHIDA, H.; DOLAN, N. J.; HOUNSOME, C.; ALAJMI, N.; BISHOP, N. C. Salivary SIgA responses to acute moderate-vigorous exercise in monophasic oral contraceptive users. TT - Appl physiol nutr metab., v. 40, n. 9 , p. 863-867, 2015.

HEJAZI, K.; HOSSEINI, S. R. A. Influence of selected exercise on serum immunoglobulin, testosterone and cortisol in semi-endurance elite runners. Asian J Sports Med, v. 3, p. 185-92, 2012. 
HIRABARA, S. M. et al. Molecular targets related to inflammation and insulin resistance and potential interventions. J Biomed Biotechnol., v. 2012, n. Figure $1,2012$.

HOFFMAN-GOETZ, L.; ZAJCHOWSKI, S. In vitro apoptosis of lymphocytes after exposure to levels of corticosterone observed following submaximal exercise. J Sports Med Physical Fitness, v. 39, p. 269-274, 1999.

HOFFMAN-GOETZ, L.; ZAICHOWSKI, S.; ALDRED, A. et al. Impact of treadmill exercise on early apoptotic cells in mouse thymus and spleen. Life Sci, v. 64, p. 161-200, 1998.

HYVÖNEN, M. T.; SPALDING, K. L. Maintenance of white adipose tissue in man. Int $\mathbf{J}$ Biochem Cell Biol., v. 56, p. 123-132, 2014.

KANDA, N.; TSUCHIDA, T.; TAMAKI, K. Testosterone inhibits immunoglobulin production by human peripheral blood mononuclear cells. Clin Exp Immunol , v. 106, p. 410-415, 1996.

KIPPELEN, P.; FITCH, K. D.; ANDERSON, S. D.; BOUGAULT, V.; BOULET, L. P.; RUNDELL, K. W.; SUECHU, M.; MCKENZIE, D. C. Respiratory health of elite athletes - preventing airway injury: a critical review. Br J Sports Med., v. 46, n. 7, p. 471-476, 2012.

KJAER, M.; DELA, F. Endocrine responses to exercise. Exercise and Immune Function, edited by L. HoffmanGoetz. Boca Raton, FL: CRC, 1996, p. 1-20.

KOPF, M.; ABEL, B.; GALLIMORE, A.; CARROLL, M.; BACHMANN, M. F. Complement component C3 promotes T-cell priming and lung migration to control acute influenza virus infection. Nat. Med. 8, 373-378, 2002.

KRÜGER, K.; AGNISCHOCK, S.; LECHTERMANN, A.; TIWARI, S.; MISHRA, M.; PILAT, C.; WAGNER, A.; TWEDDELL, C.; GRAMLICH, I.; MOOREN, F. C. Intensive resistance exercise induces lymphocyte apoptosis via cortisol and glucocorticoid receptor-dependent pathways. J Appl. Physiol. n. 23, p. 1226-1232, 2011.

KUROWSKI, M.; JURCZYK, J.; JARZEBSKA, M.; MOSKWA, S.; MALOWSKA, J. S.; KRYSTOFIAK, H.; KOWALSKI,
M. L. Association of serum Clara cell protein CC16 with respiratory infections and immune response to respiratory pathogens in elite athletes. Respir res., v. 15, p. $45,2014$.

LANCASTER, G.; HALSON, S. L.; KHAN, Q.; DRYSDALE, P.; WALLACE, F.; JEUKENDRUP, A. E.; DRAYSON, M. T.; GLEESON, M. Effects of acute exhaustive exercise and chronic exercise training on type 1 and type $2 \mathrm{~T}$ lymphocytes. Exerc Immunol Rev, v. 10, p. 91-104, 2004.

LECAILLE, F.; LALMANACH, G.; ANDRAULT, P. M. Antimicrobial proteins and peptides in human lung diseases: A friend and foe partnership with host proteases. Biochimie, 2015.

LEE, J. K.; LUCHIAN, T.; PARK, Y. Effect of Regular Exercise on Inflammation Induced by Drug-resistant Staphylococcus aureus 3089 in ICR mice. . Sci Rep., v. 5, n. April, p. 16364, 2015.

VAN DE WEERT-VAN LEEUWEN, P. B.; ARETS, H. G.; VAND DER ENT, C. K.; BEEKMAN, J. M. Infection, inflammation and exercise in cystic fibrosis. Respir res., v. 14, n. 1, p. $1,2013$.

LIU, Y. MEI, J.; GONZALES, L.; YANG, G.; DAI, N.; WANG, P.; ZHANG, P.; FAVARA, M.; MALCOLM, K. C.; GUTTENTAG, S.; WORTHEN, G. S. IL-17A and TNF- $\alpha$ exert synergistic effects on expression of CXCL5 by alveolar type II cells in vivo and in vitro. J immunol. (Baltimore, Md.: 1950), v. 186, n. 5, p. 3197-3205, 2011.

LLOPIS, P. Q.; REIG GARCÍA-GALBIS, M. Control glucémico a través del ejercicio físico en pacientes con diabetes mellitus tipo 2; revisión sistemática GLYCEMIC CONTROL THROUGH PHYSICAL EXERCISE IN TYPE 2 DIABETES SYSTEMATIC REVIEW. Nutr Hosp.Nutr Hosp, v. 3131, n. 4, p. 1465-1472, 2015.

MAISEL, A. S.; HARRIS, T.; REARDEN, C. A.; MICHEL, M. C. Betaadrenergic receptors in lymphocyte subsets after exercise. Alterations in normal individuals and patients with congestive heart failure. Circulation 82: 2003-2010, 1990.

MARTIN, L.; VAN MEEGERN, A.; DOEMMING, S.; SCHUERHOLZ, T. Antimicrobial Peptides in Human 
Sepsis. FFront Immunol., v. 6, n. August, p. 1-7, 2015.

MARTIN, S. A.; PENCE, B. D.; WOODS, J. A. Exercise and Respiratory Tract Viral Infections. Exerc Sport Sci Rev. v. 37, n. 4, p. 157-164, 2009.

MARTÍNEZ, ALFREDO CÓRDOVA; ALVAREZĐMON, M. O sistema imunológico (I): Conceitos gerais, adaptação ao exercício físico e implicações clínicas / Immunological system: general concepts, exercise adaptation and its clinical implications. Rev bras med esporte, v. 5, n. 3, p. 120-125, 1999

MATHUR, N.; PEDERSEN, B. K. Exercise as a Mean to Control Low-Grade Systemic Inflammation. Mediat inflamm, v. 2008, p. 1-6, 2008.

MCLELLAN, K. C. P.; BARBALHO, S. M.; CATTALINI, M.; LERARIO, A. C. Diabetes mellitus do tipo 2, síndrome metabólica e modificação no estilo de vida. Rev Nutr, v. 20, n. 5, p. 515-524, 2007.

MESSAGE, S. D.; JOHNSTON, S. L. Host defense function of the airway epithelium in health and disease: clinical background. J. Leukoc. Biol. v. 75, n. January, p. 5-17, 2004 .

MISHARIN, A. V.; CHI, M.; BUDINGER, G. R. S. Modeling human influenza infection in the laboratory. Infection and Drug Resistance, p. 311-320, 2015.

MORGADO, J. M.; RAMA, L.; SILVA, I.; INÁCIO, M. J.; HENRIQUES, A.; LARANJEIRA, P.; PEDREIRO, S.; ROSADO, F.; ALVES, F.; GLEESON, M.; PAÍS, M. L.; PIVA, A.; TEIXEIRA, A. M. Cytokine production by monocytes, neutrophils, and dendritic cells is hampered by long-term intensive training in elite swimmers. Eur J Appl Physiol, v. 112, n. 2, p. 471-482, 2012.

NEILSON, H. K.; FRIEDENREICH, C. M.; BROCKTON, N. T.; MILLIKAN, R. C. Physical activity and postmenopausal breast cancer: Proposed biologic mechanisms and areas for future research. Cancer epidemiol biomark prev, v. 18, n. 1, p. 11-27, 2009.

NISHIDA, Y.; TANAKA, K.; HARA, M.; HIRAO, N.; TANAKA, H.; TOBINA, T.; IKEDA, M.; YAMATO, H.; OHTA, M. Effects of home-based bench step exercise on inflammatory cytokines and lipid profiles in elderly Japanese females: A randomized controlled trial. Arch Gerontol Geriatr., v. 61, n. 3, p. 443-451, 2015.

PARK, H.; SHIN, J. W.; PARK, S. G.; KIM, W. Microbial communities in the upper respiratory tract of patients with asthma and chronic obstructive pulmonary disease. PloS one, v. 9, n. 10, p. e109710, 2014.

PARRA-SÁNCHEZ, J.; MORENO-JIMENEZ, M.; NICOLA, C. M.; NOCUA-RODRIGUEZ, I. I.; AMEGLÓ-PAREJO, M. R.; CARMEN-PEÑA, M.; CORDERO-PRIETO, C.; GAJARDOBARRENA, M. J. Evaluación de un programa de ejercicio físico supervisado en pacientes sedentarios mayores de 65 años con diabetes mellitus tipo 2. Atención Primaria, n. 20 , p. 1-8, 2015.

PEDERSEN, B. K. Muscles and their myokines. J Experimental Biology, v. 214, p. 337-346, 2011.

PEDERSEN, B. K.; HOFFMAN-GOETZ, L. Exercise and the Immune System: Regulation, Integration and Adaptation IMMUNE SYSTEM. Physiol rev., v. 80, n. 3, p. 1055-1081, 2000 .

RANTALA, A. K.; JAAKKOLA, M. S.; MAKIKYRO, E. M. S.; HUGG, T. T.; JAAKKOLA, J. J. K. Early Respiratory Infections and the Development of Asthma in the First 27 Years of Life. Am j epidemiol., p. kwv093, 2015.

RINGSEIS, R.; MOOREN, F.; KRÜGER, K. Metabolic signals and innate immune activation in obesity and exercise Exerc Immunol Rev., v. 21, p. 58-68, 2015.

RISØY, B. A.; RAASTAD, T.; HALLEN, J.; LAPPEGARD, K. T.; BAEVERFJORD, K.; KRAVDAL, A.; SIEBKE, M.; BENESTAD, H. B. Delayed leukocytosis after hard strength and endurance exercise: aspects of regulatory mechanisms. BMC physiology, v. 3, p. 14, 2003.

RODRIGUES DA CONCEIÇÃO, R.; SIMÃO, R.; SILVEIRA, A. L. B.; SILVA, G. C.; NOBRE, M.; SALERNO, V. P.; NOVAES, J. Section II-Exercise Physiology \& Sports Medicine Acute Endocrine Responses to Different Strength Exercise Order in Men. J Hum Kinet., v. 44, n. 44, p. 111-120, 2014.

RONDON, M. U. P.; BRUM, P. C. Exercício físico como tratamento não-farmacológico da hipertensão arterial. 
Rev bras hipertens., v. 10, n. 11, p. 134-139, 2003.

SAGUN, G.; OGUZ, A.; KARAGOZ, E.; FILIZER, A. T.; TAMER, G; MESCI, B. Application of alternative anthropometric measurements to predict metabolic syndrome. Clinics, v. 69, n. 5, p. 347-353, 2014.

SATURNI, S.; CONTOLI, M.; SPANEVELLO, A.; PAPI, A. Models of Respiratory Infections: Virus-Induced Asthma Exacerbations and Beyond. Allergy Asthma Immunol Res., v. 7, n. 6, p. 525-533, 2015.

SHEN, Z.; FANG, L.; ZHAO, L.; LEI, HAN. $\beta$-Defensin 2 ameliorates lung injury caused by Pseudomonas infection and regulates proinflammatory and anti-inflammatory cytokines in rat. Int J Mol Sci., v. 15, n. 8, p. 1337213387, 2014.

VAN CRAENENBROECK, A. H.; VAN ACKEREN, K.; HOYMANS, V. Y.; ROEYKENS, J.; VERPOOTEN, G. A.; VRINTS, C. J.; COUTTENYE, M. M.; VAN CRAENENBROECK, E. M. Acute Exercise-Induced Response of Monocyte Subtypes in Chronic Heart and Renal Failure. Mediat inflamm., v. 2014, p. 1-11, 2014.

VANDINI, S.; BOTTAU, P.; FALDELLA, G.; LANARI, M. Immunological, Viral, Environmental, and Individual Factors Modulating Lung Immune Response to Respiratory Syncytial Virus. BioMed Res Internat., v. 2015, p. 1-7, 2015.

WANG, J.; SONG, H.; TANG, X.; YANG, Y.; VIEIRA, V. J.; NIU, Y.; MA, Y. Effect of exercise training intensity on murine T-regulatory cells and vaccination response. Scandinavian J Medicine \& Scie Sports, v. 22, n. 5, p. 643-652, 2012.

WARREN, K. J.; OLSON, M. M.; THOMPSON, N. J.; CAHILL, M. L.; WYATT, T. A.; YOON, K. J.; LOIACONO, C. M.; KOHUT, M. L. Exercise Improves Host Response to Influenza Viral Infection in Obese and Non-Obese Mice through Different Mechanisms. Plos One, v. 10, n. 6, p. e0129713, 2015.

YANG, D.; BIRAGYN, A.; KWAK, L. W.; OPPENHEIM, J. J. Mammalian defensins in immunity: more than just microbicidal. Trends Immunol, v. 23, p. 291-296, 2002.
Recebido: 16 de janeiro de 2016

Revisado: 04 de abril de 2016 Aceito: 29 de abril de 2016 